\title{
Automatic Vehicle Recognition and Destruction for Military Application
}

\author{
Mugdha Deshmukh ${ }^{1}$, Prachi Gaikwad ${ }^{2}$, Tanmay Gaikwad ${ }^{3}$, Ashok Joglekar ${ }^{4}$ \\ ${ }^{1,2,3}$ B.E Student, Dept., of E\&TC Engineering, SAOE, Kondhwa, Pune, Maharashtra, India \\ ${ }^{4}$ Professor, Dept., of E\&TC Engineering, SAOE, Kondhwa, Pune, Maharashtra, India
}

\begin{abstract}
The need for automation in the field of military is on a constant rise as it promises troop protection and thus avoids unnecessary loss of lives. Today, most wars are fought using heavy vehicles, thus arises a need for a system to counter an attack from such machines. The "Automatic Vehicle Detection and Destruction System" doesn't only detect vehicles, but also identifies whether they belong to the enemy forces. In this project, we develop a system based on ARM 7 technology and IMAGE PROCESSING which will not only detect a vehicle based on imaging \& feature extraction, but its identity will also be confirmed. In case the vehicle cannot be identified, the system will communicate with the vehicle using encrypted signals. If a response signal isn't received the targeted vehicle will be destroyed. For this purpose we use RC4 technology to enable signal encryption and decryption.
\end{abstract}

Keywords: arm7, image processing, feature extraction, rc4technology, encryption, decryption.

\section{Introduction}

Conventionally, vehicle detection and identification was done manually. A soldier would have to risk his life and be present in the field while using his judgment. Accurate results though it may produce, but the process becomes long and cumbersome as the soldier has to identify an enemy vehicle and relay this information back to a control station. The proposed "Automatic Vehicle Recognition and Destruction System for Military Application" can complete the same task in a shorter span of time while at the same time it can help avoid risking of human lives. The system designed will not only detect nearby vehicles but also determine which army it belongs to, hence thereby determining whether it should be destroyed or not. This is accomplished in four stages:

1) Image acquisition

2) Processing of acquired image

3) Wireless communication

4) Destruction

The system is divided into two modules:

1) Missile module.

2) Vehicle module.

\section{Literature Survey}

In this section of paper the work that has been done in the area of video surveillance systems, followed by different approaches made for better results in object or vehicle recognition, tracking and destruction are described. Different approaches have been used to automate video surveillance systems according to the need of the users. In all the systems developed so far, feature extraction plays the most important role in the identification process. For this purpose different feature extraction algorithms have been used.

An attempt was made by researchers to develop a complete independent system for intrusion detection and tracking it until destroyed completely using image processing algorithm, microcontroller and other additional hardware [1], [2]. Features like shape and color of an object are extracted and compared with anobject in the database to decide whether the object is intruding one. Shape is detected using canny edge detection algorithm and for rotation of servo motor PMW pulses are used. Results are obtained in experimentation are satisfactory.

R. D. Gade and Prof.AnilWanaredeveloped a system of automatic detection, recognition and destruction of intrusion using image processing algorithm, microcontroller, and other supplementary hardware [3].Canny edge detection algorithm was used for shape detection. Our proposed system is made on the lines of the project by making it more secure using encryption decryption technology

The system developed by Rupali N. Wadekar, Dr. M. S. Nagmode which aims at destroying any intruding object in sensitive areas using image processing algorithm, SIFT algorithm for calculation of key-points of object, microcontroller and additional hardware[4]. Using this similar concept and adding identification of friend, or, foe feature is proposed work on the project.

\section{Methodology}

As mentioned above the system works in four stages:

- Image acquisition: In the first stage, a standard CCTV camera of resolution720X680p is used for acquiring images. The camera is mounted on a platform such that the entire area of interest is under surveillance. The camera position is fixed in order to avoid accuracy problems. Images from the camera are then processed on a suitable platform. The camera used can be a night vision camera or camera with different resolutions.

- Image processing: In this stage, a computer with $1.6 \mathrm{GHz}$ dual-core Intel core i5 processor (turbo boost up to 2.7 $\mathrm{GHz}$ ) with $3 \mathrm{MB}$ shared L3 cache with memory of 4GB of 1600MHz LPDDR3 onboard memory and 128 GB PCle based flash memory. MATLAB is used as a platform for processing the image. For the purpose of identification, 


\section{International Journal of Science and Research (IJSR) \\ ISSN (Online): 2319-7064}

Index Copernicus Value (2013): 6.14 | Impact Factor (2015): 6.391

various features of the object of interest must be extracted. These basic features vehicle will help determine whether the vehicle belongs to the enemy forces or not. Features of friendly vehicles must first be stored in a database, thus whenever a new vehicle is scanned, its features are compared with the ones in the database. Irrespective of a match, the system enters a third stage wherein an encrypted message is sent to the vehicle from the control station.

- Communication: In the third stage, an encrypted signal is sent to the vehicle, requesting basic identity information. An appropriate response can only be generated by a friendly vehicle. This response consists of information which reveals the vehicle identity. If the identity is confirmed, no action will be taken and the system will go back to the first stage. If the vehicle does not respond in 5 seconds, the system will declare it as an enemy vehicle and thus attack it. This communication is accomplished by using a ZIGBEE module, an IEEE802.15.4 standard, operating at frequency of $2.4 \mathrm{GHz}$ ISM.

- Destruction: In the fourth stage, an ARM 7 microcontroller based firing system attacks the enemy vehicle by locating it. Once the system locks onto the vehicle, it fires a missile to destroy it.

The basic block diagram of this implementation of the system is as shown below. The system consists of two units namely: missile unit and vehicle unit. The missile unit exists inside the control station.

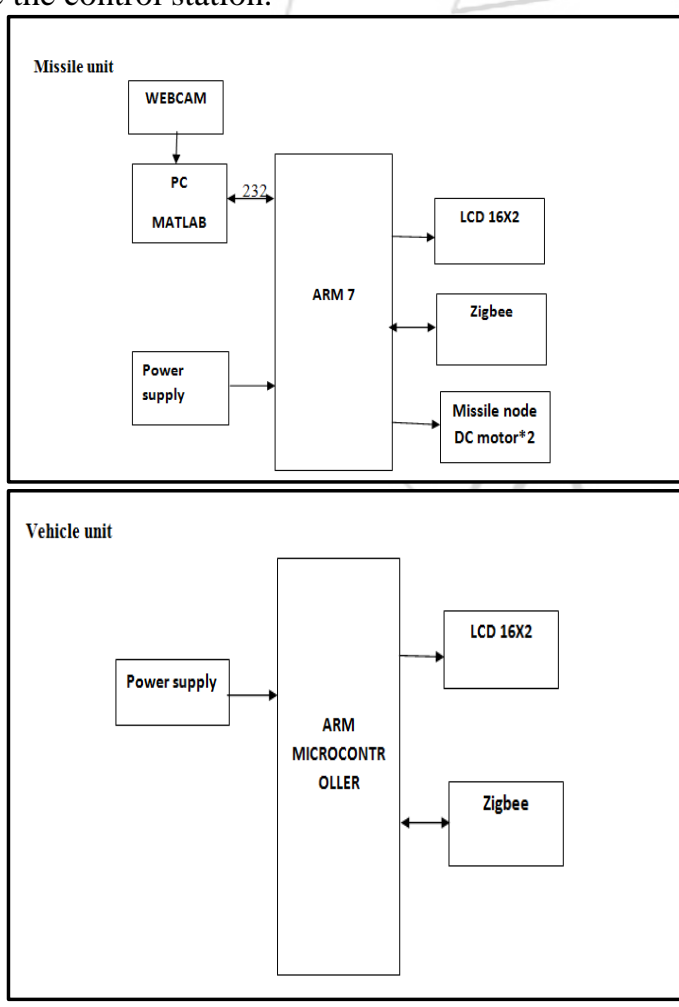

Figure 1: Block diagram of complete system

\section{Implementation}

The implementation is divided into 3 processes:

\section{1) Feature Extraction}

The most important aspect of this project is the feature extraction algorithm. For this purpose any new image is subjected to a certain amount of pre-processing. In this process the vehicle to be scanned must first be isolated i.e. separated from its background. This allows us to observe the edges of the vehicle. In order to avoid blurriness we dilate the image.Canny edge detection algorithm is used for the purpose of edge detection. Once the vehicle has been isolated we focus on the various features that have to be extracted and stored in the database:

\section{2) Colour}

The colour of the vehicle is considered to be the colour which covers the maximum vehicle surface area, e.g. if the vehicle covered with yellow colour, along with patches in black and blue colour then the vehicle is considered to be of yellow colour. To obtain the colour of the vehicle, logical ANDing of colour image acquired from camera with the preprocessed image is performed. The resulting image is converted into HSV image. The Hue plane contains only colour information. All the values of the Hue plane are in range of 0 and 1 . Colour is detected depending upon the values of HSV. E.g. red, green and yellow colours can be distinguished as in HSV plane as: Red pixels values lie in range of 0.08 to 0.15 , Green pixels values lie in range of 0.15 to 0.48 , Blue pixels values lie in range of 0.15 to 0.2 . Thus first gross feature of the vehicle that is its colour is detected.

\section{3) Shape}

In order to save shape descriptor of the vehicle, we need to calculate the distance of all the points on edge from a reference point; this point is taken as centre of mass (centroid) of the vehicle. The centre of mass does not change on rotation of vehicle. In this case, distances of points on the boundary are taken at the angles with interval of 10 degrees from centre of mass. Thus 36 different distances with 36 different angles are stored in the shape descriptor of a vehicle. In order to increase the accuracy of the system, we can decrease the interval of angles with corresponding increase in number of distances from the boundary. But this will increase the computation time. It is not necessary that in every captured frame the vehicle will be at same distance from camera. The vehicle size may vary though its shape is same. The system should work decent for different sizes of the vehicle. This is known as scale invariance. The system can be made scale invariant; by storing the distances in shape descriptor that are normalized. Normalization means division of each available distance from the maximum distance available in the descriptor. All the readings of shape descriptor lie between 0-1.1, corresponds to the maximum distance. Thus, scale invariance is achieved. Software should identify vehicle correctly even if the vehicle is rotated. This is done by circular shifting of readings. Thus, rotation invariance is achieved.

These features are first captured for a friendly vehicle and stored for the program to compare. Vehicles with the exact same features will be declared as friendly vehicles by the program; else they will be fired upon.

\section{4) Communication}

The detected vehicle is considered to be friendly based on the results of feature extraction and matching of the same with the features in database. To confirm the identity of the 


\section{International Journal of Science and Research (IJSR) \\ ISSN (Online): 2319-7064 \\ Index Copernicus Value (2013): 6.14 | Impact Factor (2015): 6.391}

detected vehicle, as a friend, the encrypted data is sent to the detected vehicle. The encryption of data is done using RC4 encryption-decryption technology. ZIGBEE RF transceiver is used in both modules, as mentioned above in the block diagram. It is connected to microcontroller of respective modules at URAT port 1 . The encrypted data, to be transmitted to the detected vehicle, is sent to microcontroller of missile module using serial communication RS232, which is then transmitted to the detected vehicle via ZIGBEE transceiver. The detected vehicle if happens to be friendly, will respond positively to encrypted data by decrypting it and the decrypted data will be sent back to missile module within stipulated time. The transmission of decrypted data is achieved using ZIGBEE.But if the detected vehicle which is considered to be friendly based on feature extraction process fails to respond positively to the encrypted data then the vehicle is declared to be enemy vehicle and further destroyed.

\section{5) Firing mechanism}

In case a response is not received by the system within the allotted time, the firing mechanism is activated. An ARM-7 LPC-2138 microcontroller is used as the controlling circuit, with an operating frequency of $60 \mathrm{MHz}$, along with a $512 \mathrm{~KB}$ of on-chip Flash user code memory. The X-Y plane position of the intruding vehicle is sent to microcontroller. The microcontroller is interfaced with a computer using a serial cable- RS232. The microcontroller sends PWM pulses to a DC motor. The angle of rotation of the DC motor is changed according to the width of the PWM pulses applied. The DC motor is in contact with the trigger for the mounted gun. As the motor rotates the trigger is pushed and the gun fires accordingly. The signal to rotate the motor is only sent when the system identifies the vehicle as an enemy vehicle. angles corresponding to 36 different distances were calculated. Once the HSV values and the out of 36 different values of distances ,if 34 values from database matches with the HSV values and 34 different distances calculated of the detected vehicle then the vehicle is recognised as a friendly vehicle else an enemy vehicle.

On recognising vehicle as friendly vehicle based on colour, shape feature, to be sure of the identity an encrypted signal is sent to the vehicle, if the vehicle is friendly it will decrypt the data and send it back to prove its identity within 5 secs. If the vehicle fails to respond to the encrypted signal in stipulated time the vehicle is destroyed using missile. But if the vehicle is not identified as friendly vehicle based on its features it is destroyed immediately. The whole process is completed in approximately $10-15$ secs.

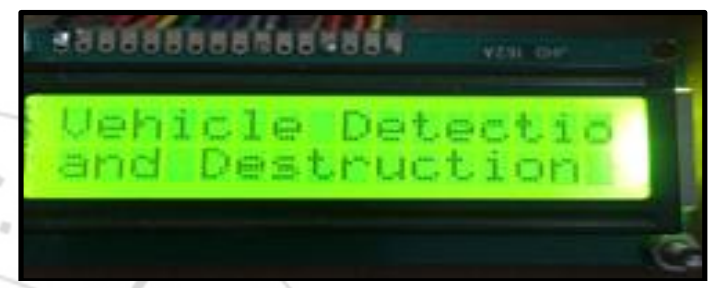

Figure 3: Initialization of the system

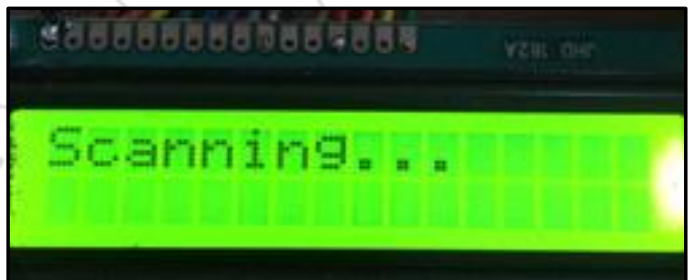

Figure 4: System scanning for vehicle

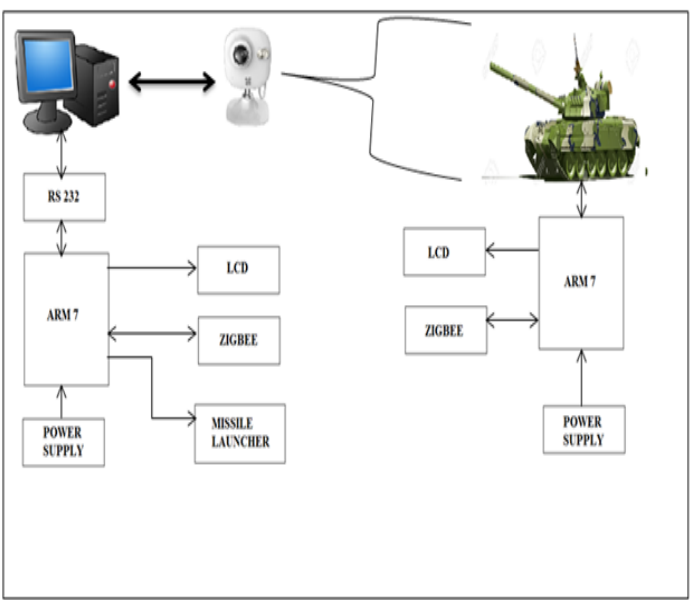

Figure 2: Functional block diagram of complete system.

\section{Experimental Results}

Two different vehicles of different size and shape were taken to create a database. For colour as a feature of identification two colours were considered one for friendly vehicle and other for enemy vehicle. Colours considered for testing are GREEN and YELLOW for friendly and enemy vehicle. For colour detection HSV values are considered. The HSV values of red colour are different from the HSV values of green and yellow colour. These values confirm the colour of vehicle. For shape determination, 36 different

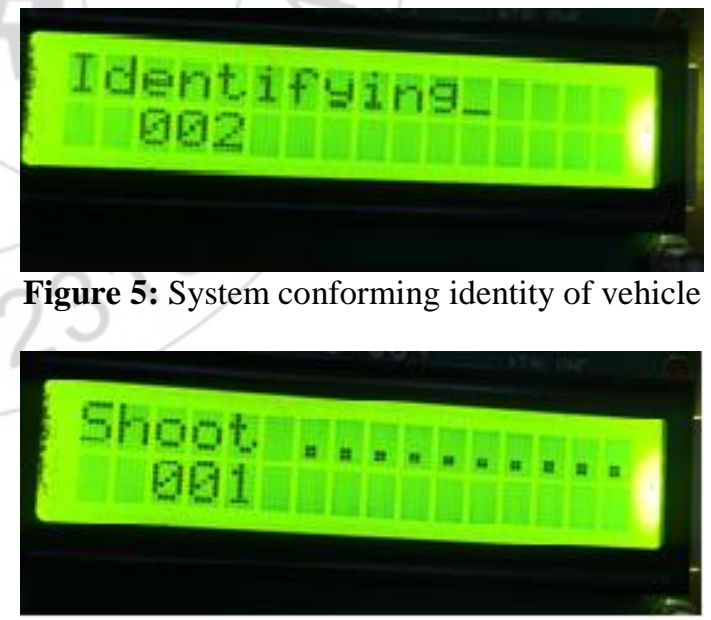

Figure 6: System destroying enemy vehicle

\section{Conclusion}

The system proposed in this paper being an automated type of surveillance and protection system, is more efficient and accurate at targeting the enemies' vehicles and convoys and protecting our friendly military bases. Also this system eliminates the use of military personnel for round the clock surveillance of the base, as the work is done automatically and thus reduces the risk of harming any friendly troop or soldier. 


\section{Future Scope}

The light source plays vital role in providing illumination to the system. Light source is available during day time (sun) but problem may occur during night time. Instead of depending on source of illumination Infrared source, thus making the system invariant. Also night vision cameras can be used. Thermal cameras provide better clarity of coloured vehicles depending on their temperature. Database can be extended by adding more number of vehicles with different shapes and sizes. Also database can be extended by adding images of the vehicles acquired at various times of the day. This will boost the accuracy of the system and make it more efficient.

\section{References}

[1] AmitKenjale, "Automatic Object Recognition, Tracking and Destruction for Military Application", World Congress on Information and Communication Technologies, pp.1485-1489, 2013.

[2] Archana R. Borse and Dilip S. Patil, "Visual Object Recognition, Tracking and Control for Automated Video Surveillance System", International Journal of Latest Research in Science and Technology, vol. 2, Issue 6, pp. 77-80, November-December 2013.

[3] R. D. Gade and Prof.AnilWanare, "Object Recognition, Tracking and Destruction for Military Application", International Journal of Advanced Engineering \& Innovative Technology (IJAEIT) Volume 1, Issue 3, August-2014 ISSN:2348-7208

[4] Rupali N. Wadekar, Dr. M. S. Nagmode, "Automatic Intrusion Recognition, Trackingand Destruction for Security Systems", International Journal of Innovative Research in Computer and Communication Engineering (An ISO 3297: 2007 Certified Organization) Vol. 3, Issue 6, June 2015. 